\title{
New Types of Q-Integral Inequalities
}

\author{
Waadallah T. Sulaiman \\ Department of Computer Engineering, College of Engineering, University of Mosul, Mosul, Iraq \\ E-mail:waadsulaiman@hotmail.com \\ Received January 13, 2011; revised March 7, 2011; accepted March 10, 2011
}

\section{Abstract}

Several new q-integral inequalities are presented. Some of them are new, One concerning double integrals, and others are generalizations of results of Miao and Qi [1]. A new key lemma is proved as well.

Keywords: Q-Integral Inequalities

\section{Introduction}

For $0<q<1$ the q-analog of the derivative, denoted by $D_{q}$ is defined by (see [2])

$$
D_{q} f(x)=\frac{f(x)-f(q x)}{x-q x}, x \neq 0 .
$$

Whenever $f^{\prime}(0)$ exists, $D_{q} f(0)=f^{\prime}(0)$, and as $q \rightarrow 1^{-}$, the q-derivative reduces to the usual derivative.

The q-analog of integration from 0 to $a$ is given by (see [3])

$$
\int_{0}^{a} f(x) \mathrm{d}_{q} x=a(1-q) \sum_{k=0}^{\infty} f\left(a q^{k}\right) q^{k}
$$

provided the sum converges absolutely. On a general interval $[a, b]$ the q-integral is defined by (see [4])

$$
\int_{a}^{b} f(x) \mathrm{d}_{q} x=\int_{0}^{b} f(x) \mathrm{d}_{q} x-\int_{0}^{a} f(x) \mathrm{d}_{q} x .
$$

The q-Jackson integral and the q-derivative are related by the fundamental theorem of quantum calculus, which can be stated as follows (see [4, p. 73]) :

If $F$ is an anti q-derivative of the function $f$, namely $D_{q} F=f$, continuous at $x=a$, then

$$
\int_{a}^{b} f(x) \mathrm{d}_{q} x=F(b)-F(a) .
$$

For any function $f$, we have

$$
D_{q} \int_{a}^{x} f(t) \mathrm{d}_{q} t=f(x)
$$

For $b>0$ and $a=b q^{n}, n \in N$, we denote

$$
[a, b]_{q}=\left\{b q^{k}: 0 \leq k \leq n\right\} \text { and }(a, b]_{q}=\left[a q^{-1}, b\right]_{q} \text {. }
$$

It is not difficult to check the following

$$
\begin{aligned}
D_{q}(f(x) g(x)) & =f(x) D_{q} g(x)+g(q x) D_{q} f(x) \\
D_{q}\left(\frac{f(x)}{g(x)}\right) & =\frac{g(x) D_{q} f(x)-f(x) D_{q} g(x)}{g(x) g(q x)}
\end{aligned}
$$

In [5] the following result was proved

Theorem 1.1. Let $f$ be a function defined on $[a, b]_{q}$ satisfying

$f(a) \geq 0$ and $D_{q} f(x) \geq(t-2)(x-a)^{t-3}$ for $x \in(a, b]_{q}$ and $t \geq 3$.

Then

$$
\int_{a}^{b} f^{t}(x) \mathrm{d}_{q} x \geq\left(\int_{a}^{b} f(q x) \mathrm{d}_{q} x\right)^{t-1}
$$

and in [1], the following results were proved

Theorem 1.2. If $f(x)$ is a non-negative and increasing function on $[a, b]_{q}$ and satisfies

$$
(\alpha-1) f^{\alpha-2}(q x) D_{q} f(x) \geq \beta(\beta-1) f^{\beta-1}(x)(x-a)^{\beta-2}
$$

for $\alpha \geq 1$ and $\beta \geq 1$ then

$$
\int_{a}^{b} f^{\alpha}(x) \mathrm{d}_{q} x \geq\left(\int_{a}^{b} f(x) \mathrm{d}_{q} x\right)^{\beta} .
$$

Theorem 1.3. If $f(x)$ is a non-negative and increasing function on $\left[b q^{n+m}, b\right]$ and satisfies

$$
(\alpha-1) D_{q} f(x) \geq \beta(\beta-1) f^{\beta-\alpha+1}\left(q^{m} x\right)(x-a)^{\beta-2}
$$

on $[a, b]_{q}$ and for $\alpha, \beta \geq 1$ then 


$$
\int_{a}^{b} f^{\alpha}(x) \mathrm{d}_{q} x \geq\left(\int_{a}^{b} f\left(q^{m} x\right) \mathrm{d}_{q} x\right)^{\beta} .
$$

Theorem 1.4. If $f(x)$ is a non-negative function on $[0, b]_{q}$ and satisfies

$$
\int_{x}^{b} f^{\beta}(t) \mathrm{d}_{q} t \geq \int_{x}^{b} t^{\beta} \mathrm{d}_{q} t
$$

for $x \in[0, b]_{q}$ and $\beta>0$ then the inequality

$$
\int_{x}^{b} f^{\beta+\alpha}(t) \mathrm{d}_{q} t \geq \int_{x}^{b} t^{\alpha} f^{\beta}(t) \mathrm{d}_{q} t
$$

holds for all positive numbers $\alpha$ and $\beta$.

Lemma 1.5[5]. Let $p \geq 1$ and $g(x)$ be a nonnegative, monotonic function on $[a, b]_{q}$.Then

$$
p g^{p-1}(q x) D_{q} g(x) \leq D_{q}\left(g^{p}(x)\right) \leq p g^{p-1}(x) D_{q} g(x) .
$$

Remark 1. It may be mentioned that the function $g$ should be non-decreasing, which is not stated. As well if $g$ is non-increasing, (16) reverses. If $g$ non-decreasing and $p$ is such that $0<p<1$, then it is not difficult to show that (14) reverses.

\section{Results}

We start with the following key lemmas

Lemma 2.1. Let $\phi, f \geq 0$, and both non-decreasing functions, $\varphi$ is differentiable, $f$ defined on $[a, b]_{q}$. Then

$\varphi^{\prime} \circ f(q x) D_{q} f(x) \leq D_{q} \phi o f(x) \leq \phi^{\prime} O f(x) D_{q} f(x),(17)$

If $f$ is non-increasing, (15) reverses.

Proof. We have

$$
\begin{aligned}
& \phi o f(x)-\phi o f(q x)=\phi(f(x))-\phi(f(q x)) \\
& =\int_{f(q x)}^{f(x)} \phi^{\prime}(t) \mathrm{d} t \leq \phi^{\prime}(f(x)) \int_{f(q x)}^{f(x)} \mathrm{d} t \\
& =\phi^{\prime}(f(x))(f(x)-f(q x))
\end{aligned}
$$

therefore

$$
\begin{aligned}
& D_{q} \phi o f(x)=\frac{\phi o f(x)-\phi o f(q x)}{(1-q) x} \\
& \leq \phi^{\prime}(f(x)) \frac{f(x)-f(q x)}{(1-q) x}=\phi^{\prime} o f(x) D_{q} f(x)
\end{aligned}
$$

The rest is also similar.

Probably the following lemma is useful in some cases.

Lemma 2.2. Let $\phi, f \geq 0$, and both non-decreasing functions, $f$ defined on $[a, b]_{q}$ Define

$$
D_{q}(\phi, f)=\frac{\phi o f(x)-\phi o f(q x)}{f(x)-f(q x)} .
$$

Then

$$
D_{q}(\phi, f) D_{q} f(x) \leq\left(\phi^{\prime} \circ f\right)(x) D_{q} f(x)
$$

Proof. We have,

$D_{q} \phi(f(x))=\frac{\phi(f(x))-\phi(f(q x))}{(1-q) x}$
$=\frac{\phi o f(x)-\phi o f(q x)}{f(x)-f(q x)} \frac{f(x)-f(q x)}{(1-q) x}=D_{q}(\phi, f) D_{q} f(x)$

By (17),

$D_{q}(\phi, f) D_{q} f(x)=D_{q} \phi o f(x) \leq\left(\phi^{\prime} o f\right)(x) D_{q} f(x)$.

All the rest are similar.

Theorem 2.3. Let $\phi, \varphi, f, g \geq 0, \varphi, g$ are both nondecreasing and defined on $[a, b]_{q}, \varphi \circ g(a)=0$. If

$$
(\phi \circ f)(x) \geq\left(\varphi^{\prime} \circ g\right)(x) D_{q} g(x),
$$

then

$$
\int_{a}^{x}(\phi o f)(t) \mathrm{d}_{q} t \geq(\varphi \circ g)(x) .
$$

If $g$ is non-increasing and

$$
(\phi \circ f)(x) \geq\left(\varphi^{\prime} \circ g\right)(q x) D_{q} g(x),
$$

satisfies, then (21) reverses.

Proof. Set

$$
F(x)=\int_{a}^{x}(\phi o f)(t) \mathrm{d}_{q} t-(\varphi \circ g)(x) .
$$

We have, by lemma 2.1,

$$
\begin{aligned}
& D_{q} F(x)=D_{q}\left(\int_{a}^{x}(\phi \circ f)(t) \mathrm{d}_{q} t\right)-D_{q}(\varphi \circ g)(x) \\
& \geq(\phi \circ f)(x)-\left(\varphi^{\prime} \circ g\right)(x) D_{q} g(x) \geq 0 .
\end{aligned}
$$

Therefore, $F$ is non-decreasing, which implies $F(x) \geq F(a)=0$.

The result follows.

Corollary 2.4. Let $f(x)$ be a nonnegative and increasing function on $[a, b]_{q}$ such that $f(a)=0$. Let $\alpha>\gamma>0, \alpha \geq 1, \beta \geq 2$. If

$$
\begin{aligned}
& (\alpha-\gamma) f^{\alpha-\gamma-1}(q x) D_{q} f(x) \\
& \geq \beta(\beta-1) f^{\gamma(\beta-1)}(x)(x-a)^{\beta-2},
\end{aligned}
$$

is satisfied, then

$$
\int_{a}^{b} f^{\alpha}(x) \mathrm{d}_{q} x \geq\left(\int_{a}^{b} f^{\gamma}(x) \mathrm{d}_{q} x\right)^{\beta} .
$$


Furthermore, if

$$
\begin{aligned}
& (\alpha-\gamma) f^{\alpha-\gamma-1}(q x) D_{q} f(x) \\
& \geq \beta(\beta-1) f^{\gamma(\beta-1)}(q x)(x-a)^{\beta-2}
\end{aligned}
$$

is satisfied, then

$$
\int_{a}^{b} f^{\alpha}(x) \mathrm{d}_{q} x \geq\left(\int_{a}^{b} f^{\gamma}(q x) \mathrm{d}_{q} x\right)^{\beta}
$$

Proof. For $x \in[a, b]_{q}$ let

$$
\phi(x)=x^{\alpha}, \varphi(x)=x^{\beta}, g(x)=\int_{a}^{x} f^{\gamma}(x) \mathrm{d}_{q} x
$$

then, we have, via lemma 1.5,

$$
\begin{aligned}
& (\phi \circ f)(x)-\left(\varphi^{\prime} \circ g\right)(x) D_{q} g(x) \\
& =f^{\alpha}(x)-\beta\left(\int_{a}^{x} f^{\gamma}(t) d_{q} t\right)^{\beta-1} f^{\gamma}(x) \\
& =f^{\gamma}(x)\left(f^{\alpha-\gamma}(x)-\beta\left(\int_{a}^{x} f^{\gamma}(t) d_{q} t\right)^{\beta-1}\right)=f^{\gamma}(x) h(x) .
\end{aligned}
$$

Now,

$$
\begin{aligned}
D_{q} h(x)= & D_{q} f^{\alpha-\gamma}(x)-\beta D_{q}\left(\int_{a}^{x} f^{\gamma}(t) d_{q} t\right)^{\beta-1} \\
\geq & (\alpha-\gamma) f^{\alpha-\gamma-1}(q x) D_{q} f(x) \\
& -\beta(\beta-1)\left(\int_{a}^{x} f^{\gamma}(t) d_{q} t\right)^{\beta-2} f^{\gamma}(x) \\
\geq & (\alpha-\gamma) f^{\alpha-\gamma-1}(q x) D_{q} f(x) \\
& -\beta(\beta-1) f^{\gamma(\beta-1)}(x)(x-a)^{\beta-2} \\
\geq & 0 .
\end{aligned}
$$

Therefore, $h(x)$ is non-decreasing, but $h(a)=0$, then $h(x) \geq 0$. The result follows by theorem 2.3 .

The proof of the second part is similar, and therefore, it is omitted.

Remark 2. Theorem 1.2 follows from corollary 2.4, the first part, by putting $\gamma=1$.

Theorem 2.5. Let $f, g$ are non-negative functions on $[a, b]_{q}$, either $f$ or $g$ is non-decreasing and they satisfies

$$
\int_{x}^{b} f^{\beta}(t) \mathrm{d}_{q} t \geq \int_{x}^{b} g^{\beta}(t) \mathrm{d}_{q} t, x \in[a, b]_{q},
$$

then the inequality

$$
\int_{x}^{b} f^{\alpha+\beta}(t) \mathrm{d}_{q} t \geq \int_{x}^{b} f^{\alpha}(t) g^{\beta}(t) \mathrm{d}_{q} t
$$

holds for all positive numbers $\alpha$ and $\beta$.
Proof. Suppose that $f$ is non-decreasing. Using the fact

$$
f(b)-f(a)=\int_{a}^{b} D_{q} f(x) \mathrm{d}_{q} x
$$

we have

$$
\begin{aligned}
& \int_{b}^{b} f^{\alpha+\beta}(x) \mathrm{d}_{q} x=\int_{a}^{b} f^{\beta}(x)\left(\int_{a}^{x} D_{q} f^{\alpha}(t) d_{q} t+f(a)\right) \mathrm{d}_{q} x \\
& =\int_{a}^{b} D_{q} f^{\alpha}(t) \int_{t}^{b} f^{\beta}(x) d_{q} x d_{q} t+f(a) \int_{a}^{b} f^{\beta}(x) \mathrm{d}_{q} x \\
& \geq \int_{a}^{b} D_{q} f^{\alpha}(t) \int_{t}^{b} g^{\beta}(x) d_{q} x d_{q} t+f(a) \int_{a}^{b} g^{\beta}(x) \mathrm{d}_{q} x \\
& =\int_{a}^{b} g^{\beta}(x)\left(\int_{a}^{x} D_{q} f^{\alpha}(t) d_{q} t+f(a)\right) \mathrm{d}_{q} x \\
& =\int_{a}^{b} f^{\alpha}(x) g^{\beta}(x) \mathrm{d}_{q} x .
\end{aligned}
$$

Now, suppose $g$ is non-decreasing, then, we have

$$
\begin{aligned}
& \int_{b}^{b} f^{\beta}(x) g^{\alpha}(x) \mathrm{d}_{q} x \\
& =\int_{a}^{b} f^{\beta}(x)\left(\int_{a}^{x} D_{q} g^{\alpha}(t) \mathrm{d}_{q} t+g(a)\right) \mathrm{d}_{q} x \\
& =\int_{a}^{b} D_{q} g^{\alpha}(t) \int_{t}^{b} f^{\beta}(x) \mathrm{d}_{q} x \mathrm{~d}_{q} t+g(a) \int_{a}^{b} f^{\beta}(x) \mathrm{d}_{q} x \\
& \geq \int_{a}^{b} D_{q} g^{\alpha}(t) \int_{t}^{b} g^{\beta}(x) \mathrm{d}_{q} x \mathrm{~d}_{q} t+g(a) \int_{a}^{b} g^{\beta}(x) \mathrm{d}_{q} x \\
& =\int_{a}^{b} g^{\beta}(x)\left(\int_{a}^{x} D_{q} g^{\alpha}(t) \mathrm{d}_{q} t+f(a)\right) \mathrm{d}_{q} x \\
& =\int_{a}^{b} g^{\alpha+\beta}(x) \mathrm{d}_{q} x .
\end{aligned}
$$

Using the arithmetic geometric inequality yields

$$
\frac{\beta}{\alpha+\beta} f^{\alpha+\beta}(x)+\frac{\alpha}{\alpha+\beta} g^{\alpha+\beta}(x) \geq f^{\beta}(x) g^{\alpha}(x) \text {. }
$$

Integrating the above inequality and making use of (29) gives

$$
\begin{aligned}
& \frac{\beta}{\alpha+\beta} \int_{a}^{b} f^{\alpha+\beta}(x) \mathrm{d} x+\frac{\alpha}{\alpha+\beta} \int_{a}^{b} g^{\alpha+\beta}(x) \mathrm{d} x \\
& \geq \int_{a}^{b} f^{\beta}(x) g^{\alpha}(x) \mathrm{d} x \geq \int_{a}^{b} g^{\alpha+\beta}(x) \mathrm{d} x .
\end{aligned}
$$

The result follows.

Remark 3. Theorem 1.4 follows from theorem 2.5 by putting $a=0, g(x)=x$.

Corollary 2.6. Let $f \geq 0$. If 


$$
\frac{\sin f(x)}{f(x)} \geq \cos \left(\int_{a}^{x} f(t) \mathrm{d}_{q} t\right), x \in[a, \pi / 2]_{q}
$$

then

$$
\int_{a}^{x} \sin (f(t)) \mathrm{d}_{q} t \geq \sin \left(\int_{a}^{x} f(t) \mathrm{d}_{q} t\right)
$$

Proof. The proof follows from theorem 2.3 by putting

$$
\phi(x)=\varphi(x)=\sin x, g(x)=\int_{a}^{x} f(t) \mathrm{d}_{q} t,
$$

as follows

$$
\begin{aligned}
& (\phi \circ f)(x)-\left(\varphi^{\prime} \circ g\right)(x) D_{q} g(x) \\
& =\sin (f(x))-\cos \left(\int_{a}^{x} f(t) d_{q} t\right) f(x) \geq 0 .
\end{aligned}
$$

The following result concerning similar inequality but for double integrals.

Theorem 2.7. Let $f \geq 0$ non-decreasing in both $x$ and $y, \quad f(a, y)=0, \alpha>\beta \gamma, \quad \beta \geq 2, \gamma>0$. If

$$
\begin{aligned}
& f^{\alpha-\beta \gamma-1}(q x, y) D_{q, x} f(x, y) \\
& \geq \frac{\beta(\beta-1)}{(\alpha-\beta \gamma)}(x-a)^{\beta-2}(y-a)^{\beta}, x, y \in[a, b]_{q},
\end{aligned}
$$

then

$$
\int_{a}^{x} \int_{y}^{b} f^{\alpha}(u, v) \mathrm{d}_{q} u \mathrm{~d}_{q} v \geq\left(\int_{a}^{x} \int_{a}^{y} f^{\gamma}(u, v) \mathrm{d}_{q} u \mathrm{~d}_{q} v\right)^{\beta}
$$

\section{Proof. Set}

$$
F(x, y)=\int_{a}^{x} \int_{y}^{b} f^{\alpha}(u, v) \mathrm{d}_{q} u \mathrm{~d}_{q} v-\left(\int_{a}^{x} \int_{a}^{y} f^{\gamma}(u, v) \mathrm{d}_{q} u \mathrm{~d}_{q} v\right)^{\beta} .
$$

We have via lemma 2.1 and by keeping $y$ fixed,

$$
\begin{gathered}
D_{q, x} F(x, y)=D_{q, x} \int_{a}^{x} \int_{y}^{b} f^{\alpha}(u, v) \mathrm{d}_{q} u \mathrm{~d}_{q} v \\
-D_{q, x}\left(\int_{a}^{x} \int_{a}^{y} f^{\gamma}(u, v) \mathrm{d}_{q} u \mathrm{~d}_{q} v\right)^{\beta} \\
D_{q, x} F(x, y) \geq \int_{y}^{b} f^{\alpha}(x, v) \mathrm{d}_{q} v
\end{gathered}
$$

$$
\begin{aligned}
& -\beta\left(\int_{a}^{x} \int_{a}^{y} f^{\gamma}(u, v) \mathrm{d}_{q} u \mathrm{~d}_{q} v\right)^{\beta-1} \int_{a}^{y} f^{\gamma}(x, v) \mathrm{d}_{q} v \\
\geq & f^{\alpha}(x, y)(b-y) \\
& -\beta f^{\gamma(\beta-1)}(x, y)(x-a)^{\beta-1}(y-a)^{\beta-1} f^{\gamma}(x, y)(y-a) \\
= & f^{\beta \gamma}(x, y)\left((b-y) f^{\alpha-\beta \gamma}(x, y)-\beta(x-a)^{\beta-1}(y-a)^{\beta}\right) \\
= & f^{\beta \gamma}(x, y) k(x) .
\end{aligned}
$$

Now,

$$
\begin{aligned}
D_{q, x} k(x) \geq & (\alpha-\beta \gamma)(b-y) f^{\alpha-\beta \gamma-1}(q x, y) D_{q} f(x, y) \\
& -\beta(\beta-1)(x-a)^{\beta-2}(y-a)^{\beta} \\
\geq & 0 .
\end{aligned}
$$

Therefore, $k$ is non-decreasing, as $k(a)=0$ then $k(x) \geq 0$ which implies $D_{q, x} F(x, y) \geq 0$. that is $F(x, y)$ is non-decreasing in $x$. But $F(a, y)=0$, then $F(x, y) \geq 0$.

\section{References}

[1] Y. Miao and F. Qi, "Several Q-Integral Inequalities," Journal of Mathematical Inequalities, Vol. 3, No. 1, 2009, pp. 115-121.

[2] E. W. Weisstein, "q-derivative," From Math World - A Wolfram Web Resource, 2010.

http://math-world.wolfram.com/q-Deriative.html

[3] E. W. Weisstein, "q-Integral," Math World-A Wolfram Web Resource, 2010. http://mathworld. wolfram.com/q-Integral.html

[4] V. Kac and P. Cheung, "Quantum Calculus," "Universitex, Springer-Verlag, New York, 2002. doi:10.1007/978-1-4613-0071-7

[5] K. Brahim, N. Bettaibi and M. Sellemi, "On Some Feng Qi Type q-Integral Inequalities," Journal of Inequalities in Pure and Applied Mathematics, Vol. 9, No. 2, 2008, Art. 43. 